\title{
Maintaining momentum for collaborative working groups in a post-pandemic world
}

To the editor - Scientific progress depends in part on our ability to synthesize heterogeneous data and ideas into new models and paradigms. In environmental sciences, such synthesis has been particularly effective when conducted by collaborative working groups: diverse groups of researchers and practitioners brought together for a concentrated period of collaboration on key questions. Such work is often done at synthesis centres: organizations that promote, fund, organize and host working groups and other collaborative research and training activities $^{1}$. However, because of the COVID19 pandemic, synthesis centres have had to rapidly adapt to supporting fully virtual working groups; the eight centres we direct supported 68 virtual working groups in the past year. Based on this experience, we conclude - contrary to a recent editorial on conferences published in this journal ${ }^{2}$ - that virtual gatherings, while providing a bridge during the pandemic, cannot replace immersive, in-person collaborations. In-person working group meetings involve productive and varied interactions for many hours over consecutive days. We have found that virtual sessions lose effectiveness after a few hours, as participants become fatigued from staring at a screen or juggling local demands. While virtual meetings can work for short, well-delineated tasks, they are less suited for unstructured and free-flowing discussions - and thus struggle to create the social cohesion and trust known to fuel creative breakthroughs during week-long in-person meetings ${ }^{3}$.

The transition from in-person to entirely virtual working groups was abrupt. By contrast, we envision a gradual reverse transition to in-person collaboration, requiring hybrid models as a temporary bridge in the period when public health measures relax and travel becomes possible again. But beyond this transition period, some elements of hybrid models may also have lasting utility for improving productivity, inclusiveness or outreach. Hybrid models use strong virtual collaborations to link in-person meetings over space or time, including expanding the participant pool at the proposal or outreach stages (Fig. 1). During a period when international travel remains restricted but local travel and meetings may be safe,

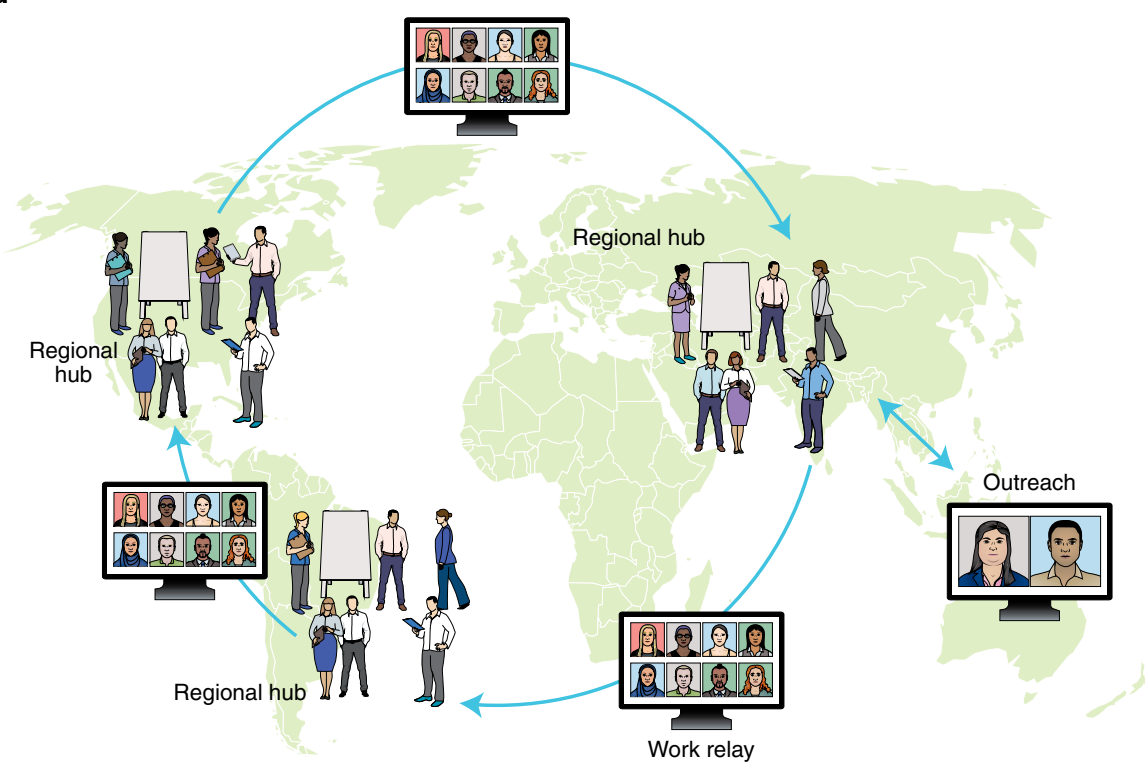

b

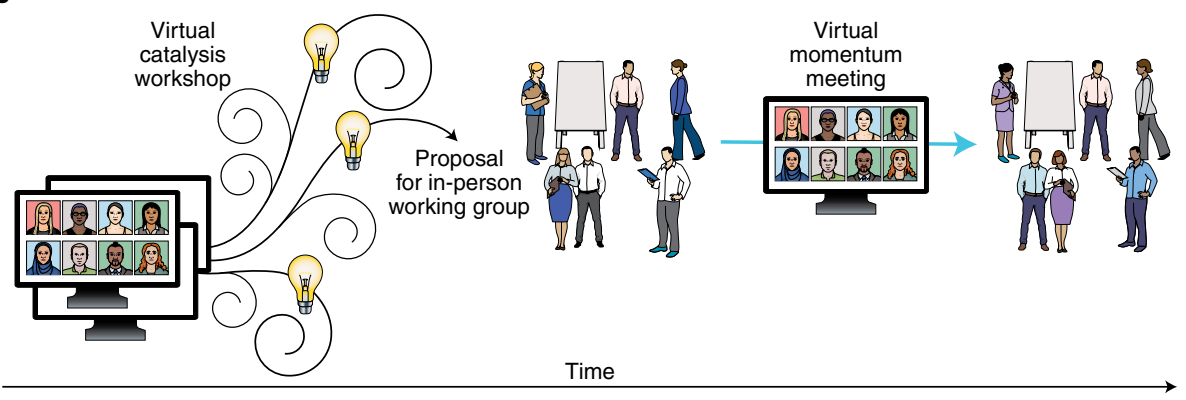

Fig. 1 | Two ways that virtual collaboration can augment but not replace in-person working groups. a, Spatial hybrid models extend regional collaborations across geographic space. In the model shown, research teams within each geographic region meet simultaneously as in-person working groups, but these 'regional hubs' also coordinate virtually with each other. One type of coordination is a 'work relay' where a team in one time zone hands off a task to a team in a different time zone whose workday is just beginning. Virtual communication is also useful for 'outreach': connecting working groups with knowledge providers and end users of the science. $\mathbf{b}$, Temporal hybrid models enrich the flow of the working group process. In 'virtual catalysis workshops', large numbers of participants brainstorm potential questions for a future in-person working group. This hybrid model makes the process of forming questions more inclusive, while leveraging the power of in-person collaboration for the solution process. Interspersing in-person working group meetings with short 'virtual momentum meetings' can maintain the impetus.

distributed regional teams might meet in person but coordinate virtually with each other (including focused one-on-one conversations). Such a model distributed across time zones can also allow for a type of work relay ${ }^{4}$. A regional hub model
(Fig. 1a) could even reinforce cooperation between synthesis centres and be the basis of joint opportunities involving international funding agencies (for example, Belmont Forum, Future Earth and BiodivERsA). This hybrid approach may eventually transition 
to a model that overcomes funding limits, increases global integration and reduces aeroplane-related carbon emissions.

Complementary aspects of in-person and virtual collaboration can emerge at different points in the working group dynamic. Virtual collaborations allow large numbers of diverse participants. This can be useful in idea-generating or 'catalysis' workshops, used by several synthesis centres to democratize the proposal development stage by involving a wide range of researchers and practitioners. Virtual versions of catalysis workshops (Fig. 1b) can improve diversity by including participants whose personal or professional commitments limit their ability to travel. For example, childcare and high teaching loads can prevent travel, and such gendered responsibilities likely reduced the scientific productivity of women during the pandemic ${ }^{5}$. However, virtual interactions should not be seen as a way to reduce costs. As trust is required for effective virtual brainstorming, synthesis centres would need to invest in skilled facilitators for virtual catalysis meetings to be effective.

While the actual working group meetings are best done in person, interspersing these meetings with bursts of virtual interactions can keep projects moving forward (virtual momentum meetings, Fig. 1b). In-person working groups can also engage virtually with individuals who face tight time constraints (for example, policymakers) or limited mobility (for example, elders), ensuring that the science being developed incorporates different types of knowledge and meets the needs of end users (outreach, Fig. 1a). Although some of these hybrid models are already encouraged by synthesis centres, we envision more widespread use given the increased comfort with video conferencing in both academic and non-academic spheres.
Embracing such hybrid models can help synthesis centres and similar organizations be even more impactful and relevant than before the pandemic. They will, however, require new investment by synthesis centres in international cooperative agreements and skilled personnel to facilitate this navigation between physical and virtual worlds.

Working groups supported by synthesis centres play an outsized role in fostering the collective advancement and application of science $e^{1,6}$. Such working groups not only produce seminal syntheses of existing results, but ignite new areas of research with novel and interdisciplinary concepts ${ }^{6}$. This has been brought into stark relief by the pandemic, as working groups struggle to recreate the special social ingredients online that make them so productive in person. We envision a future where virtual interactions, instead of replacing in-person working groups, usefully build on the strengths of such collaboration. By using the best of virtual and social worlds, working groups can still let the inspiration of the unplanned fuel the realization of the planned.

Diane S. Srivastava (D) 1凶, Marten Winter (D)2,3, Louis J. Gross (D) 4,5, Jean Paul Metzger (D),7, Jill S. Baron ${ }^{8}$, Nicolas Mouquet (D) 9,10,

Thomas R. Meagher (D) 11,

Benjamin S. Halpern (D) 12,13 and

Valério D. Pillar (D) 7,14

${ }^{1}$ Canadian Institute of Ecology and Evolution, Biodiversity Research Centre, University of British Columbia, Vancouver, British Columbia, Canada. ${ }^{2}$ German Centre for Integrative Biodiversity Research (iDiv) Halle-Jena-Leipzig, Leipzig, Germany. ${ }^{3}$ Leipzig University, Leipzig, Germany. ${ }^{4}$ National Institute for Mathematical and Biological Synthesis (NIMBioS), University of Tennessee, Knoxville, TN, USA. ${ }^{5}$ Departments of Ecology and Evolutionary Biology and Mathematics, University of Tennessee, Knoxville, TN, USA. ${ }^{6}$ Department of Ecology,
Institute of Biosciences, University of São Paulo, São Paulo, Brazil. ${ }^{7}$ Synthesis Centre on Biodiversity and Ecosystem Services (SinBiose), Brasília, Brazil. ${ }^{8} \mathrm{John}$ Wesley Powell Center for Analysis and Synthesis, US Geological Survey, Fort Collins, CO, USA. ${ }^{9}$ Centre for the Synthesis and Analysis of Biodiversity (CESAB), Fondation pour la recherche sur la biodiversité, Montpellier, France. ${ }^{10}$ MARBEC, Univ Montpellier, CNRS, Ifremer, IRD, Montpellier, France. ${ }^{11}$ Centre for Biological Diversity, School of Biology, University of St Andrews, St Andrews, UK. ${ }^{12}$ National Center for Ecological Analysis and Synthesis, University of California, Santa Barbara, CA, USA. ${ }^{13}$ Bren School of Environmental Science and Management, University of California, Santa Barbara, CA, USA. ${ }^{14}$ Department of Ecology, Universidade Federal do Rio Grande do Sul, Porto Alegre, Brazil.

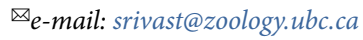

Published online: 13 July 2021

https://doi.org/10.1038/s41559-021-01521-0

References

1. Baron, J. S. et al. 67, 750-759 (2017).

Nat. Ecol. Evol. 4, 1569 (2020).

3. Hackett, E., Parker, J., Conz, D., Rhoten, D. \& Parker, A. In Scientific Collaboration on the Internet (eds Olson, G. et al. 277-296 (MIT Press, 2008).

4. Halpern, B. S. et al. Ecology and COVID \#4: Leveraging the liabilities of virtual collaboration. ESA https://go.nature. com/3dofzxX (3 June 2020).

5. King, M. M. \& Frederickson, M. E. Socius 7, 1-24 (2021).

6. Halpern, B. S. et al. Bioscience 70, 1005-1014 (2020).

Acknowledgements

The authors acknowledge valuable discussion with other members of the International Synthesis Consortium, including M. de Araujo Mamede, M. Palmer, J. Kramer, R. Beilinson, J. Arnott and P. Kille. J.B. thanks USGS internal reviewers K. Bagstad and W. Sanford. We also acknowledge all synthesis group members who have been incredibly creative in continuing their research dynamic despite going $100 \%$ virtual. M.W. acknowledges funding by the DFG (via iDiv: FZT 118, 02548816). Any use of trade, firm, or product names is for descriptive purposes only and does not imply endorsement by the US Government.

Competing interests

The authors declare no competing interests. 